\title{
Emergence of High Level Azithromycin-Resistant Neisseria gonorrhoeae Strain Isolated in Argentina
}

\author{
Patricia G. Galarza, MSC, MPH, *† Belén Alcalá, PHD, $\neq$ Celia Salcedo, PHD, \\ Liliana Fernández Canigia, MSC,§ Luis Buscemi, MSC,II Irene Pagano, MD, $\dagger$ \\ Claudia Oviedo, BSC, $\dagger$ and Julio A. Vázquez, PHD
}

\begin{abstract}
One Neisseria gonorrhoeae strains highly resistant to azithromycin AzHLR (MIC $>2048 \mathrm{mg} / \mathrm{L}$ ) was isolated in Argentina in 2001 and it has been characterized by $N$. gonorrhoeae multiantigen sequence typing (NG-MAST) as ST696, suggesting a different event to other isolates in Europe. Neither, mtrR mutations or presence of mef gene were detected.
\end{abstract}

Key Words: Azithromycine resistance, Gonococci, mtrR

Sinterting ingle oral dose $(1 \mathrm{~g})$ of azithromycin is a recommended therapy for chlamydial infections. However, this dose is not enough effective for gonorrhea and because the use of $2 \mathrm{~g}$ (highly effective again Neisseria gonorrhoeae) has been reported as being associated with gastrointestinal intolerance, is not widely recommended for gonorrhea. ${ }^{1}$ However, several countries might have been using this antibiotic both for the primary treatment of gonococcal infections and for the simultaneous treatment of chlamydial infections, ${ }^{2}$ and it is strongly recommended to monitor gonococcal resistance to azithromycin in countries where this drug is used. ${ }^{1}$

The National Reference Center for STDs in Argentina include azithromycin since 1997 on the routine surveillance susceptibility patterns for gonococcal strains isolated in hospitals belonging to the National Gonococcal Susceptibility Surveillance Program. As result of this, 2 azithromycin-resistant gonococcal strains were detected over the period between 1997and 2004: the first one in November 2001 and the second one in May 2003, with MICs of $>2048$ and $8 \mathrm{mg} / \mathrm{L}$, respectively. ${ }^{3}$ Susceptibility to azithromycin was determined by the agar dilution method according with the CLSI guidelines, ${ }^{4}$ using 0.03 to $2048 \mathrm{mg} / \mathrm{L}$ as range of concentrations, and was confirmed by the Spanish Reference Laboratory for Neisseria, using both agar dilution and Etest method.

From the *Centro Nacional de Referencia en Enfermedades de Transmisión Sexual (CNR-ETS), Instituto Nacional de Enfermedades Infecciosas-ANLIS "Dr. Carlos G. Malbrán," Buenos Aires, Argentina; Instituto Nacional de Enfermedades Infecciosas-ANLIS "Dr. Carlos G. Malbrán,” Buenos Aires, Argentina; †Laboratorio de Referencia de Neisseria, Centro Nacional de Microbiología, Instituto de Salud Carlos III, Madrid, Spain; $\S \ddagger$ Servicio de Microbiologia, Hospital Alemán, Buenos Aires, Argentina; and q[Servicio de Microbiologia, Hospital Muñiz, Buenos Aires, Argentina

Correspondence: Patricia G. Galarza, MSc, MPH, Servicio de Enfermedades de Transmisión Sexual (ETS). Centro Nacional de Referencia en ETS, Departamento de Bacteriología. Instituto Nacional de Enfermedades Infecciosas A.N.L.I.S. "Dr Carlos G. Malbrán." Avda Vélez Sarsfield 563, 1281 Buenos Aires, Argentina. E-mail: pgalarza@anlis.gov.ar

Received for publication April 1, 2009, and accepted June 14, 2009.

DOI: 10.1097/OLQ.0b013e3181b61bb1

Copyright @ 2009 American Sexually Transmitted Diseases

Association

All rights reserved.
Recently the emergence of high-level azithromycin resistance (AzHLR; >256 mg/L) in gonococci has been described in Scotland ${ }^{5}$ and England and Wales. ${ }^{6}$ In Scotland, they have been isolated since 2004 and most of them belong to either the sequence type (ST) 470 or the ST649. In England and Wales, a cluster of six AzHL isolates was reported all of them belonging to the ST649. The gonococcal strain with AzHLR isolated in Argentina was characterized by $N$. gonorrhoeae multiantigen sequence typing ${ }^{7}$ (NG-MAST) as belonging to the ST696 by sequencing internal regions of the genes encoding 2 variable outer membrane proteins, $\operatorname{Por}$ and $\operatorname{TbpB}$ (available at: http://www.ng-mast.net). The 3 mentioned STs (ST470, ST649, and ST696) present a common $T b p B$ allele (29) but 3 different Por sequences (339, 442, and 105 respectively). The Por alelle 105 present in the Argentinean strain shows 27 and 28 bases pair difference with Por 339 and 442, respectively, indicating different genetic lineages and suggesting independent events to produce gonococcal strains with AzHLR both in Latin America and Europe. The long passed time between the descriptions of this type of strains might suggest not easy transmission and/or survival for this type of strains. However, the finding of a cluster of isolates from heterosexual patients in England and Wales and the continuous increase notice in Scotland most probably show a real potential for spreading.

The Argentinean strain with an MIC to azithromycin of $8 \mathrm{mg} / \mathrm{L}$ showed a new ST with a Por/TbpB allelic combination of 1389/4. Both strains, with azithromycin resistance (MICs of 8 and $>2048 \mathrm{mg} / \mathrm{L}$ ) were characterized as auxotype/serovar classes Proline-/IByust and Proline-/IBrpyst, respectively, using the methodology already described. ${ }^{8,9}$ The analysis of both strains by Pulsed Field Gel Electrophoresis (PFGE) using BglII (data not showed) presented two different and unrelated pattern profiles ( 7 band differences), concluding that both gonococci belong to 2 different clones that might have acquired the resistance in 2 different and independent genetic events.

Decreased susceptibility to azithromycin in N. gonorrhoeae is mainly due, to an overproduction of mtr(CDE)encoded efflux pump determined by mtrR mutations. ${ }^{7}$

These changes have been described affecting different sites, reflected on Table 1 . For the characterization of the mechanism for resistance in both Argentinean gonococcal strains with azithromycin resistance, 2 gonococci with intermediate resistance (MIC $1 \mathrm{mg} / \mathrm{L}$ ) and 1 susceptible strain (MIC $0.06 \mathrm{mg} / \mathrm{L}$ ), all of them isolated in Argentina, were also included (Table 1).

The $m t r R$ sequence of the strain with AzHLR ( $\left.{ }^{\circ} 1782\right)$ showed one of the alterations already associated with decreased susceptibility to azithromycin. The strain $n^{\circ} 2498$ with an MIC of $8 \mathrm{mg} / \mathrm{L}$ presented a dinucleotide (TT) insertion which has been also described previously. ${ }^{10}$ Finally, those gonococcal strains (2516 and 2518) showing intermediate resistance to azithromycin appeared with changes that have been also asso- 
TABLE 1. Analysis of Changes in the mtrR Gene Associated With Decreased Susceptibility to Azithromycin on the Gonococcal Strains Included in This Study

\begin{tabular}{lcclcl} 
Strain $\mathrm{N}^{\circ}$ & MIC mg/L & Year of Isolation & City of Isolation & Gly-45 K $\rightarrow$ Asp-45 & $\begin{array}{c}\text { Inverted Repeat } \\
\text { Region }\end{array}$ \\
\hline 1782 & $>2048$ & 2001 & Buenos Aires & + & - \\
2498 & 8 & 2003 & Buenos Aires & - & - \\
2516 & 1 & 2003 & Neuquén & - & - \\
2518 & 1 & 2003 & El Palomar & - & - \\
3783 & 0.06 & 2005 & Resistencia & -
\end{tabular}

ciated with this level of resistance. Those mutations appearing on strains 2498 and particularly 1782 did not explain the high level of resistance to this antibiotic, because they have been already described in strains only showing intermediate level of resistance.

The mef gene code for an efflux pump conferring also resistance to macrolides, particularly in Gram-positive but also described in Gram-negative species. ${ }^{11}$ The presence of the mef gene was checked by PCR either in the strain 1782 and 2498 and none of them presented the gene.

Mutations in the peptidyl transferase loop in domain $\mathrm{V}$ of 23S rRNA have been also associated with macrolide resistance in $N$. gonorrhoeae. ${ }^{12}$ To identify mutations within domain V (of the peptidyltransferase loop) for each of the 4 copies of the 23S rRNA gene, a 2-step PCR method is ongoing for checking the presence of mutations in Argentinean strains showing azithromycin resistance.

Further studies are in progress trying to characterize the mechanisms for high level of resistance to azithromycin in these gonococcal strains.

The very high MIC levels recorded here would make the suggestion of using new formulations of azithromycin, ${ }^{13}$ showing less problems of gastrointestinal intolerance untenable, if strains of this type were to emerge elsewhere and spread. Decreased susceptibility to azithromycin in Latin America and the Caribbean region has been already detected through GASP network surveillance ${ }^{14}$ and, particularly in Argentina, gonococcal strains showing an MIC of $1 \mathrm{mg} / \mathrm{L}$ to azithromycin ranged from 9 to $17 \%$ during 1999 to 2001, and an average of 5.6\% until 2006, falling to $2.8 \%$ in 2007. Besides the 2 strains presented in this study, 3 additional isolates with MICs of 16 $\mathrm{mg} / \mathrm{L}$ were detected in 2004 from different provinces ${ }^{15}$ and 1 of $2 \mathrm{mg} / \mathrm{L}$ in each year during 2004, 2005, and 2006. The fall in incidence of azithromycin resistance might be reflecting either a success in recommendations to restrict the use of the antibiotic or a temporal and natural change in the prevalence of these types of strains. Otherwise, the finding of isolates with high level of resistance proves the relevance of azithromycin susceptibility surveillance in order to define the real utility of this antibiotic for the treatment of gonococcal infections in Argentina.

\section{REFERENCES}

1. Tapsall JW, Shultz TR, Limnios EA, et al. Failure of azithromycin therapy in gonorrhea and discorrelation with laboratory test parameters. Sex Transm Dis 1998; 25:505-508.
2. Dillon JA, Rubabaza JP, Benzaken AS, et al. Reduced susceptibility to azithromycin and high percentages of penicillin and tetracycline resistance in Neisseria gonorrhoeae isolates from Manaus, Brazil, 1998 Sex Transm Dis 2001; 28:521-526.

3. Clinical and Laboratory Standards Institute. Performance Standards for Antimicrobial Susceptibility Testing. Seventeenth Informational Supplement. Wayne, PA: CLSI; 2007. CLSI document M100-S17.

4. Galarza P, Alcalá B, Fernández Canigia F, et al. Emergence of high level azithromycin resistant Neisseria gonorrhoeae strains isolated in Argentina. Presented at the16th Biennial meeting of the international society for sexually transmitted diseases research (ISSTDR); July 10-13, 2005; Amsterdam, The Netherlands.

5. Palmer HM, Young H, Winter A, et al. Emergence and spread of azithromycin-resistant Neisseria gonorrhoeae in Scotland. J Antimicrob Chemother 2008; 62:490-494.

6. Chisholm SA, Ison C. Emergence of high-level azithromycin resistance in Neisseria gonorrhoeae in England and Wales. Euro Surveill 2008; 13. pii:18832.

7. Martin IC, Ison C, Aanensen DM, et al. Rapid sequence-based identification of gonococcal transmission clusters in a large metropolitan area. J Infect Dis 2004; 189:1497-1505.

8. Fiorito S, Galarza P, Pagano I, et al. Emergence of high level ciprofloxacin resistant Neisseria gonorrhoeae strain in Buenos Aires, Argentina. Sex Transm Infect 2001; 77:77.

9. Alcala B, Arreaza L, Salcedo C, et al. Molecular characterization of ciprofloxacin resistance of gonococcal strains in Spain. Sex Transm Dis 2003; 30:395-398.

10. Zarantonelli L, Borthagaray G, Lee EH, et al. Decreased susceptibility to azithromycin and erythromycin mediated by a novel $m t r(\mathrm{R})$ promoter mutation in Neisseria gonorrhoeae. J Antimicrob Chemother 2001; 47:651-654.

11. Luna VA, Cousin S Jr, Whittington WL, et al. Identification of the Conjugative mef gene in clinical Acinetobacter junii and Neisseria gonorrhoeae isolates. Antimicrob Agents Chemother 2000; 44:2503-2506.

12. Ng LK, Martin I, Liu G, et al. Mutation in 23S rRNA associated with macrolide resistance in Neisseria gonorrhoeae. Antimicrob Agents Chemother 2002; 46:3020-3025.

13. Workowski KA, Berman SM, Douglas JM Jr. Emerging antimicrobial resistance in Neisseria gonorrhoeae: urgent need to strengthen prevention strategies. Ann Intern Med 2008; 148:606613.

14. Sosa J, Ramirez-Arcos S, Ruben M, et al. High percentages of resistance to tetracycline and penicillin and reduced susceptibility to azithromycin characterize the majority of strain types of Neisseria gonorrhoeae isolates in Cuba, 1995-1998. Sex Transm Dis 2003; 30:443-448.

15. Mendez E, Morano S, Mollerach A, et al. Vigilancia de la resistencia de Neisseria gonorrhoeae en un hospital de la provincia de Santa Fe, Argentina: 1997-2004. Rev Arg Microbiol 2008; 40:173-179. 